\title{
Inhibition of Magnaporthe oryzae by Culture Filtrates of Fungi Isolated from Wild Mushrooms
}

\author{
Quyet Nguyen Thi, Kana Ueda, Junichi Kihara, Makoto Ueno* \\ Faculty of Life and Environmental Science, Shimane University, Matsue, Japan \\ Email: ${ }^{*}$ makoto-u@life.shimane-u.ac.jp
}

Received 6 August 2015; accepted 6 September 2015; published 9 September 2015

Copyright (C) 2015 by authors and Scientific Research Publishing Inc.

This work is licensed under the Creative Commons Attribution International License (CC BY).

http://creativecommons.org/licenses/by/4.0/

(c) (i) Open Access

\begin{abstract}
Fungal strains isolated from the fruiting bodies of wild mushrooms were evaluated for fungicidal activity against Magnaporthe oryzae, the causal agent of the rice blast disease. Fungal isolates $(\mathrm{n}=$ 105) were obtained from 46 samples of wild mushrooms. Infection behaviors of $M$. oryzae were assessed in the presence of culture filtrates from 90 fungal isolates, of which 20 inhibited spore germination. Heat-treated culture filtrates of these isolates were classified into 3 groups according to biological activity. Blast lesion formation by $M$. oryzae was significantly inhibited by pretreatment with culture filtrates from 4 fungal isolates. ITS region sequence analysis indicated that these isolates shared similarities with species of the genera Annulohypoxylon, Nigrospora, and Penicillium. Studies of symbiotic and parasitic fungi from wild mushrooms may yield potential control agents for plant diseases such as the rice blast disease.
\end{abstract}

\section{Keywords}

Rice Blast Disease, Culture Filtrate, Antifungal Compound, Magnaporthe oryzae, Mushroom

\section{Introduction}

Rice is one of the world's most important food crops and the rice blast disease caused by Magnaporthe oryzae (Hebert) Barr is the most destructive fungal disease affecting global rice production. Also, M. oryzae causes blast disease on several grass species including the cereals wheat and barley.

Resistant rice cultivars and chemical fungicides are important in blast disease control. However, the durability of genetic resistance in improved rice cultivars is often short-lived in the field because the pathogen rapidly evolves to overcome resistance [1]. The key to chemical control of rice blast disease is to overcome fungicide-

\footnotetext{
*Corresponding author.
}

How to cite this paper: Thi, Q.N., Ueda, K., Kihara, J. and Ueno, M. (2015) Inhibition of Magnaporthe oryzae by Culture Filtrates of Fungi Isolated from Wild Mushrooms. Advances in Microbiology, 5, 686-692.

http://dx.doi.org/10.4236/aim.2015.510071 
resistant pathogens [2] [3]. Therefore, a search for antifungal compounds is required to develop new fungicides. Antifungal compounds of microbial origin play an important role in the biological and chemical control of plant diseases [4]-[6].

Some phylloplane and endophytic fungi protect plants from pathogens. These fungi produce antimicrobial compounds [7]-[9]. Kawamata et al. (2004) [10] reported that fungal species isolated from the phylloplane of rice plants inhibit not only $M$. oryzae conidium germination and mycelial growth but also blast lesion formation. Furthermore, Pseudomonas aeruginosa (Schroeter) Migula isolated from spent mushroom substrate significantly inhibits Pyricularia grisea (Cooke) Sacc, the causal agent of gray leaf spot in perennial ryegrass [11].

The number of mushroom species in the world is estimated to be about 140,000 , but only $10 \%$ have been identified [12]. There are no reports on the existence of symbiotic and parasitic fungi in wild mushrooms and their utility in pathogen control. The objectives of this study were to: 1) isolate symbiotic and parasitic fungi from the fruiting bodies of wild mushrooms; 2) investigate the antifungal activity of culture filtrates of these fungi against M. oryzae; and 3) investigate the protective effect of the culture filtrates of the fungal isolates on lesion formation by M. oryzae in barley leaves.

\section{Materials and Methods}

\subsection{Isolation of Fungi from Fresh Fruiting Bodies of Wild Mushrooms}

Fungi were isolated from fresh fruiting bodies of 46 wild mushroom samples collected from a paddy field in Fukui, Hiroshima, Kumamoto, Osaka, Shimane, and Tottori prefectures in 2009-2011. Each fresh fruiting body was cut with a blade. Segments (approx. $5 \times 5 \mathrm{~mm}$ ) from each fresh fruiting body were removed and surface-sterilized in $80 \%$ ethanol for 2 min and 1\% sodium hypochlorite solution for 3 min, then rinsed twice in sterile distilled water for $2 \mathrm{~min}$. Rinsed segments were placed on sterile filter paper in petri dishes to remove excess surface water. After drying the segments, each segment was placed on potato sucrose agar (PSA) medium containing chloramphenicol $(200 \mu \mathrm{g} / \mathrm{mL})$ in petri dishes $(9 \mathrm{~cm}$ diameter) and incubated for 14 days at $27^{\circ} \mathrm{C}$. Fungal isolates that grew on the segments were transferred to fresh PSA medium and fungal isolates obtains by single spore or colony was maintained on PSA slants until use.

\subsection{Preparation of the Culture Filtrate}

Mycelial disks (7 mm diameter) prepared from a single colony of each isolate grown on PSA medium for 7 days were incubated in a test tube containing $20 \mathrm{~mL}$ potato sucrose broth (PSB). The culture was kept in the dark condition at $27^{\circ} \mathrm{C}$ and shaken at $120 \mathrm{rpm}$ for 10 days using rotary shaker. Culture filtrates were collected through a $0.2-\mu \mathrm{m}$ membrane filter.

\subsection{Infection-Inhibiting Activity of the Culture Filtrates}

Magnaporthe oryzae (strain Naga 69 - 150, race 007) was grown on rice bran agar at $26^{\circ} \mathrm{C}$ for 14 days, washed with running water to remove aerial hyphae, and kept at $26^{\circ} \mathrm{C}$ under near-ultraviolet radiation (FL20s BL-B; Panasonic, Osaka, Japan) for 2 days to induce sporulation.

M. oryzae spores $\left(1 \times 10^{5}\right.$ spores $\left./ \mathrm{mL}\right)$ were suspended in the fungal culture filtrates, distilled water or PSB were dropped onto glass slides and kept in a moist chamber at $26^{\circ} \mathrm{C}$. After $24 \mathrm{~h}$ incubation, the percentage of spore germination was determined by light microscopy. Data analyses were performed by using the Scheffe's test.

\subsection{Heat Pretreatment}

Selected culture filtrates were heated at $121^{\circ} \mathrm{C}$ for $20 \mathrm{~min}$ using the autoclave. The treated and untreated filtrates were inoculated together with a spore suspension $\left(1 \times 10^{5} \mathrm{spores} / \mathrm{mL}\right)$ of $M$. oryzae onto glass slides and kept in a moist chamber at $26^{\circ} \mathrm{C}$. After $24 \mathrm{~h}$ incubation, the percentages of spore germination and appressorium formation were determined by light microscopy. Data analyses were performed by using the Scheffe's test.

\subsection{Culture Filtrate Pretreatment and Fungal Inoculation}

To investigate the effects of culture filtrates on the induction of blast resistance, barley seedlings (Hordeumvul- 
gare L., cv. Minoriomugi) at the 1- to 2-leaf stage were sprayed with culture filtrates. PSB and distilled water spray were used as a control. The pretreated barley plants were kept under light condition in a moist chamber at $26^{\circ} \mathrm{C}$ for $24 \mathrm{~h}$, and then sprayed with a suspension of $M$. oryzae spores $\left(1 \times 10^{5} \mathrm{spores} / \mathrm{mL}\right)$. Inoculated plants were incubated in a moist chamber at $26^{\circ} \mathrm{C}$. Blast lesions on barley leaves were counted 4 days after inoculation.

\subsection{DNA Extraction and PCR Amplification and Sequencing}

To identify the active isolate, the sequence of the internal transcribed spacer (ITS) region (including 5.8S rDNA) was determined by PCR with the ITS1 (5'-TCCGTAGGTGAACCTGCGG-3') and ITS4 (5'-TCCTCCGCT TATTGATATGC-3') primers. Fungal genomic DNA was extracted from the mycelia as described by Suzuki et al. (2006) [13], and used as the PCR template. PCR amplification of the ITS region was performed as follows: an initial step of $5 \mathrm{~min}$ at $95^{\circ} \mathrm{C}$; followed by 30 cycles of denaturation at $94^{\circ} \mathrm{C}$, for 1 min, annealing at $62^{\circ} \mathrm{C}$, for $1 \mathrm{~min}$, and elongation at $72^{\circ} \mathrm{C}$, for $1 \mathrm{~min}$; and a final step for $5 \mathrm{~min}$ at $72^{\circ} \mathrm{C}$. PCR-amplified fragments were purified by using the HiYield Gel/PCR DNA fragment extraction kit (RBC Bioscience, Taipei, Taiwan). DNA sequencing was performed with a Big Dye Terminator Cycle Sequencing Kit (Applied Biosystems, Carlsbad, CA, USA). DNA sequence analysis was performed on an ABI PRIZM 3130xl Genetic Analyzer (Applied Biosystems, Carlsbad, CA, USA). Sequence homology was determined by searching with the BLAST suite of programs (DNA Data Bank, Japan).

\subsection{Statistical Analysis}

Data are reported as mean \pm standard deviation. Significant differences from the values were determined using a Scheffe's test $(p<0.05)$.

\section{Results and Discussion}

105 fungal isolates were obtained from the 46 fruiting bodies of wild mushrooms collected. Twenty of 90 fungal culture filtrates inhibited $M$. oryzae spore germination. The percent spore germination after treatment with isolates F5, K7, K24, K36, O1, S5, and S8 was showed 1.8\% \pm 5.3\%, 12.4\% \pm 21.0\%, 34.7\% \pm 35.5\%, 18.7\% $\pm 7.3 \%$, $2.9 \% \pm 5.9 \%, 0.9 \% \pm 2.0 \%$, and $27.3 \% \pm 19.2 \%$, respectively. On the other hand, the percent spore germination of culture filtrates by using of isolates of F1, F2, F3, H2, H5, H7, H9, K1, K9, K21, O3, O6, and S9 was $0 \%$. Germination in the distilled water and PSB controls was 95.8\% $\pm 3.7 \%$ and $90.9 \% \pm 5.7 \%$ (Figure 1).

The heated-culture filtrates $(n=20)$ were classified into 3 groups according to their inhibitory activity. The first group (F3, H2, K21, K24, and S8) showed no antifungal activity (Figure 2). These culture filtrates did not inhibit $M$. oryzae infection behaviors such as spore germination and appressorium formation. The second group (F2, F5, H9, K1, O1, O3, O6, S5, and S9) inhibited M. oryzae infection behaviors. The third group (F1, H5, H7, K7, K9, and K36) inhibited appressorium formation but not spore germination (Figure 2). Ethyl acetate extracts of culture filtrate of isolates F2, F5, H9, K1, O1, O3, O6, S5, and S9 significantly inhibited both spore germination and appressorium formation. However, F3, H2, K21, K24, and S8 did not (Figure 2). Some fungi produce enzymes such as chitinase and $\beta$-1,3-glucanase that mediate cell wall and cell membrane damage in plant pathogens [14] [15]. Our results suggested that the antifungal compound(s) in the culture filtrates of F2, F5, H9, $\mathrm{K} 1, \mathrm{O} 1, \mathrm{O} 3$, O6, S5, and S9 were not high-molecular-weight enzymes such as chitinase and $\beta$-1,3-glucanase. However, F3, H2, K21, K24, and S8 may express chitinase and $\beta$-1,3-glucanase. F1, H5, H7, K7, K9, and K36 may express high-molecular-weight enzymes as well as low-molecular-weight metabolites.

Barley leaves were sprayed with culture filtrates of isolates F2, F5, H9, K1, O1, O3, O6, S5, and S9, all of which show antifungal activity, and inoculated with $M$. oryzae spores $24 \mathrm{~h}$ later. Blast lesion formation was significantly inhibited by pretreatment with culture filtrates of O1, O3, S5, and S9 in comparison to distilled water and PSB controls. However, culture filtrates of F2, F5, H9, K1, and O6 did not inhibit blast lesion formation (Figure 3).

To identify the mycological characteristics of the obtained fungi, the ITS region including 5.8S rDNA was PCR-amplified from the genomic DNA of isolates O1, O3, S5, and S9 using ITS1 and ITS4 primers. The sequences shared similarity with Annulohypoxylon sp. (S9), Nigrospora sp. (S5), and Penicillium sp. (O1 and O3) (Table 1).

Penicillium spp. are known biocontrol agents with inhibitory activities against various plant pathogens. Peni- 

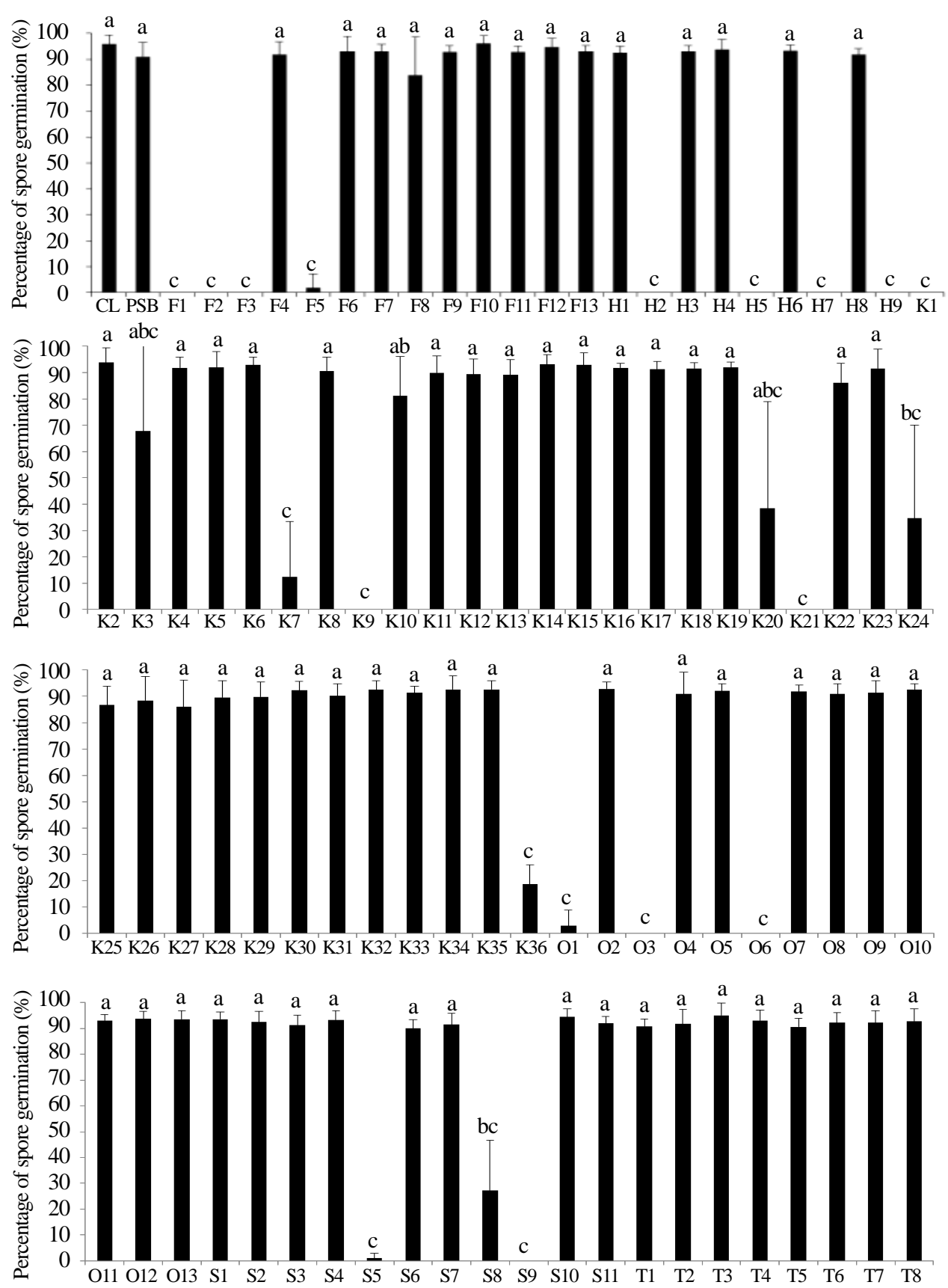

Figure 1. Culture filtrates of fungi isolated from wild mushrooms influence the infection behaviors of Magnaporthe oryzae. Data were obtained from the results of 6 experiments of 3 replicates and expressed as mean \pm SD. Means followed by different letters are significantly different according to the Scheffe's test $(p<0.05)$.

cillium spp. produce antifungal compounds such as mycophenolic acid, patulin, 3-omethylfunicone, and decumbenone A, which directly or indirectly inhibit infection by plant pathogens [16] [17]. In addition, phomalactone isolated from Nigrospora sphaerica (Sacc.) E.W. Mason inhibits mycelial growth and affects the infection behavior of plant pathogens [18]. Nigrosporin A and B, which are phytotoxic metabolites, are produced from a culture filtrate of Nigrospora oryzae (Berk. \& Br.) Petch [19]. However, in this study, the culture filtrate of Nigrospora sp. showed no phytotoxic activity in barley. There are no reports on the utility of culture filtrates of Annulohypoxylon for control of plant pathogens. Annulohypoxylon is members of the Xylariaceae family and can 


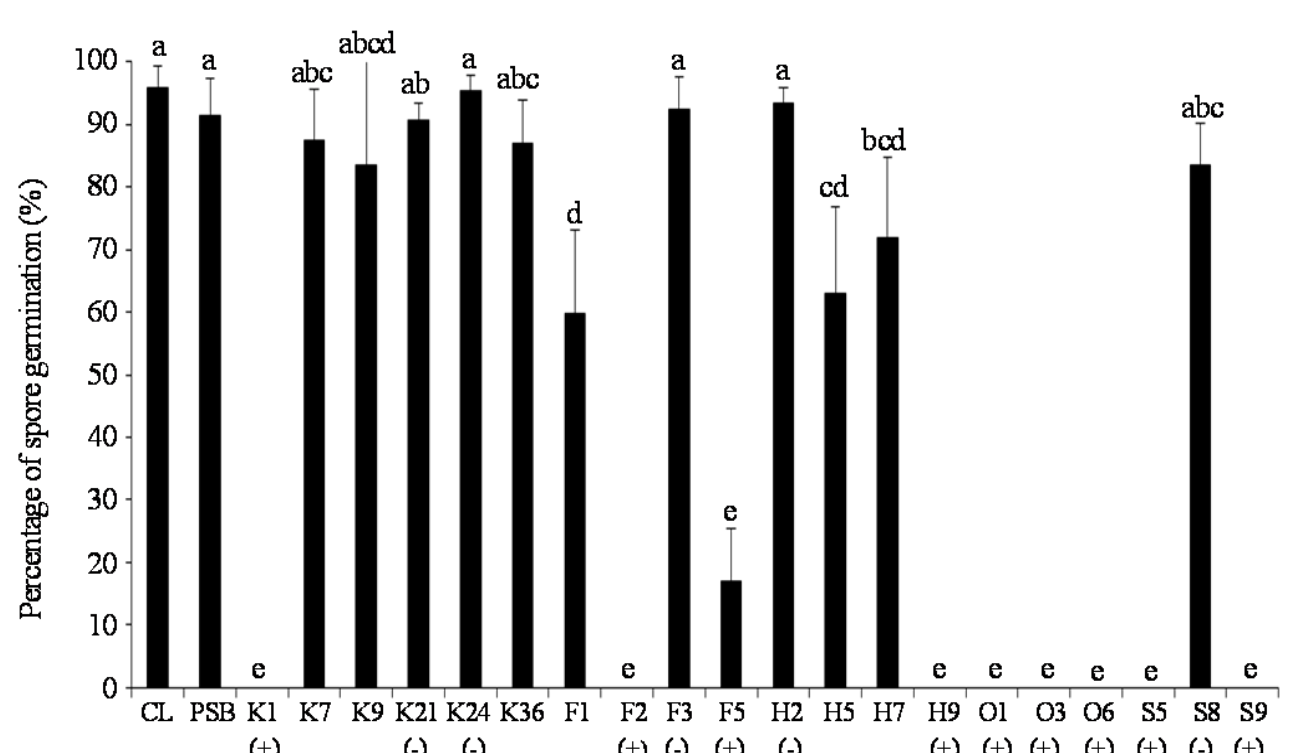

(+)

$(-)$

$(+)(-) \quad(+) \quad(-)$

$(+)(+)(+)(+)(+)(-)(+)$

(a)

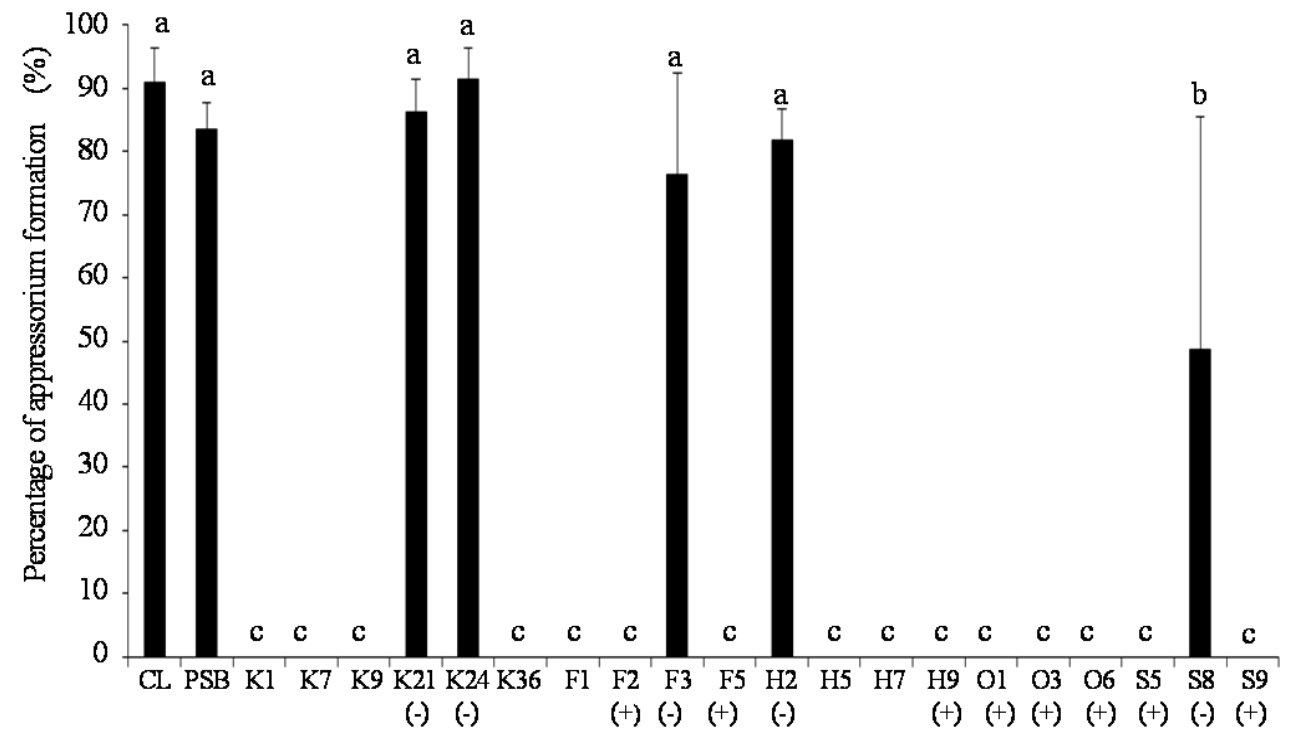

(b)

Figure 2. Inhibition by heat-treated culture isolates on the infection behaviors of Magnaporthe oryzae. (a) and appressorium formation (b) were determined after $24 \mathrm{~h}$ incubation. $(+)$ : culture filtrates with ethyl acetate soluble $(+)$ and insoluble $(-)$ antifungal compound against $M$. oryzae spore germination and appressorium formation. Data were obtained from the results of 6 experiments of 3 replicates and expressed as mean \pm SD. Means followed by different letters are significantly different according to the Scheffe's test $(p<0.05)$.

survive as endophytes. Xylariaceae is a large family (Xylariales, Ascomycotina) of more than 36 genera. Whalley (1995) [20] reported that secondary metabolites produced by representatives at least one-third of these genera have been isolated and identified. Further studies are essential to identify the active antifungal compound(s) in the culture filtrates of these fungi. Studies of symbiotic and parasitic fungi from wild mushrooms may yield potential control agents for plant diseases such as the rice blast disease.

\section{Acknowledgements}

The authors thank the faculty of Life and Environmental Science in Shimane University for help in financial 


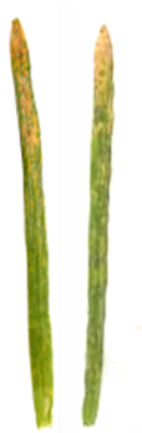

CL PSB

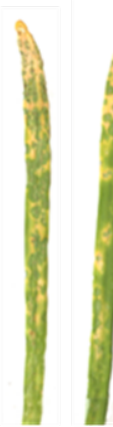

$\mathrm{F} 2$

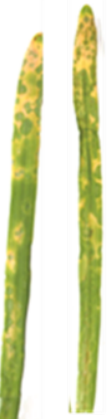

H9

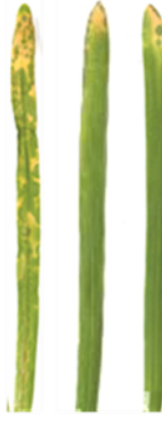

K1

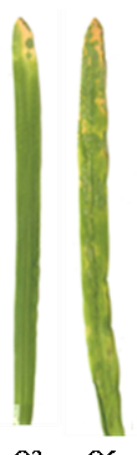

$06 \quad$ S5 $\quad$ S9

(a)

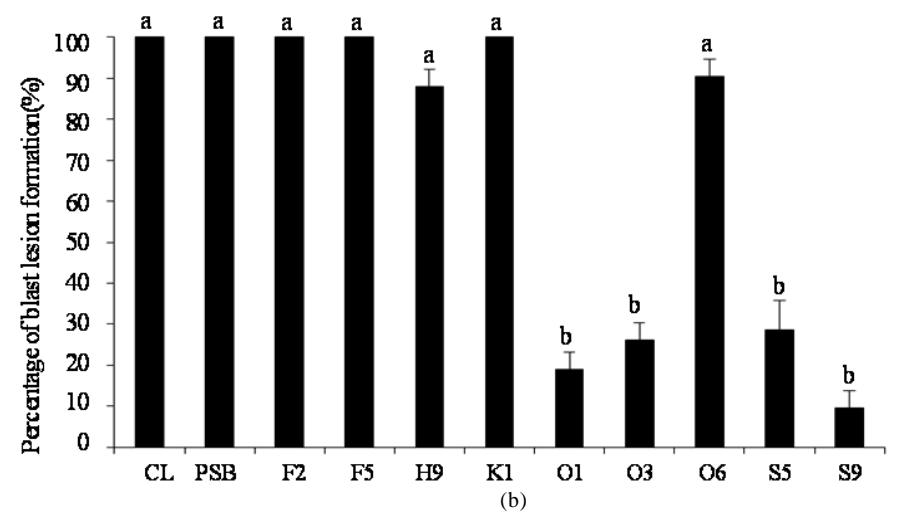

Figure 3. Inhibition by culture filtrates of selected isolates on lesion formation by Magnaporthe oryzae in barley leaves. (a) Blast lesion formation by M. oryzae. (b) Percentage of blast lesion formation by $M$. oryzae. Data were obtained from the results of 10 experiments of 3 replicates and expressed as mean \pm SD. Means followed by different letters are significantly different according to the Scheffe's test $(p<0.05)$.

Table 1. Identification of fungi isolated from mushroom by sequences of rDNA ITS regions.

\begin{tabular}{cccc}
\hline Isolate & Candidate fungus & Sampling location & Sampling year \\
\hline O1 & Penicillium sp. & Japan (Osaka) & 2010 \\
O3 & Penicillium sp. & Japan (Osaka) & 2010 \\
S5 & Nigrospora sp. & Japan (Shimane) & 2009 \\
S9 & Annulohypoxylon sp. & Japan (Shimane) & 2009 \\
\hline
\end{tabular}

supports for publishing this report.

\section{References}

[1] Ahn, S.W. (1994) International Collaboration on Breeding for Resistance to Rice Blast. In: Zeigler, R.S., Leong, S.A. and Teng, P.S., Eds., Rice Blast Disease, CAB International, Wallingford, 137-153.

[2] So, K., Fuji, M., Iwabuchi, H., Kanayama, M. and Yamaguchi, J. (2002) Effects of Various Fungicides against Less Carpropamid-Sensitive Rice Blast Fungus Isolated from the Northwest Area in Saga Prefecture. Japanese Journal of Phytopathology, 68, 262. (Abstract, in Japanese)

[3] Yamaguchi, J., Kuchiki, F., Hirayae, K. and So, K. (2002) Decreased Effect of Carpropamid for Rice Blast Control in the West North Area of Saga Prefecture in 2001. Japanese Journal of Phytopathology, 68, 261. (Abstract, in Japanese)

[4] Fravel, D.R. (1988) Role of Antibiosis in the Biocontrol of Plant Diseases. Annual Review of Phytopathology, 26, 75- 


\section{1. http://dx.doi.org/10.1146/annurev.py.26.090188.000451}

[5] Shimizu, M., Nakagawa, Y., Sato, Y., Furuma, T., Igaroshi, Y., Onaka, H., Yoshida, R. and Kunoh, H. (2000) Studies on Endophyticactinomycetes and Streptomyces sp. Isolated from Rododendron and Its Antifungal Activity. Journal of General Plant Patholology, 66, 360-366. http://dx.doi.org/10.1007/PL00012978

[6] Uddin, W. and Viji, G. (2002) Biological Control of Turfgrass Disease. In: Gnanamanickam, S.S., Ed., Biological Control of Crop Disease, CRC Press, Marcel Dekker, New York, 313-337.

[7] Aremu, E.A., Furumai, T., Igarashi, Y., Sato, Y., Akamatsu, H., Kodama, M. and Otani, H. (2003) Specific Inhibition of Spore Germination of Alternaria brassicicola by Fistupyrone from Streptomyces sp. TP-A0569. Journal of General Plant Pathology, 69, 211-217. http://dx.doi.org/10.1007/s10327-003-0038-y

[8] Evueh, G.A. and Ogbebor, N.O. (2008) Use of Phylloplane Fungi as Biocontrol Agent against Colletotrichum Leaf Disease of Rubber (Heveabrasiliensis Muell. Arg.). African Journal of Biotechnology, 15, 2569-2572.

[9] Koitabashi, M., Kajitani, Y. and Hirashima, K. (2004) Antifungal Substances Produced by Fungal Strain Kyu-W63 from Wheat Leaf and Its Taxonomic Position. Journal of General Plant Pathology, 70, 124-130. http://dx.doi.org/10.1007/s10327-003-0095-2

[10] Kawamata, H., Narisawa, K. and Hashiba, T. (2004) Suppression of Rice Blast by Phylloplane Fungi Isolated from Rice Plants. Journal of General Plant Pathology, 70, 131-138. http://dx.doi.org/10.1007/s10327-003-0100-9

[11] Viji, G., Uddin, W. and Romaine, C.P. (2003) Suppression of Gray Leaf Spot (Blast) of Perennial Ryegrass Turf by Pseudomonas aeruginosa from Spent Mushroom Substrate. Biological Control, 26, 233-243. http://dx.doi.org/10.1016/S1049-9644(02)00170-6

[12] Wasser, S.P. (2002) Medicinal Mushrooms as a Source of Antitumor and Immunomodulating Polysaccharides. Applied Microbiology and Biotechnology, 60, 258-274. http://dx.doi.org/10.1007/s00253-002-1076-7

[13] Suzuki, S., Taketani, H., Kusumoto, K.I. and Kashiwagi, Y. (2006) High-Throughput Genotyping of Filamentous Fungus Aspergillus oryzae Based on Colony Direct Polymerase Chain Reaction. Journal of Bioscience and Bioengineering, 102, 572-574. http://dx.doi.org/10.1263/jbb.102.572

[14] Lorito, M., Peterbauer, C., Hayes, C.K. and Harman, G.E. (1994) Synergistic Interaction between Fungal Cell Wall Degrading Enzymes and Different Antifungal Compounds Enhances Inhibition of Spore Germination. Microbiology, 140, 623-629. http://dx.doi.org/10.1099/00221287-140-3-623

[15] Shoresh, M. and Harman, G.H. (2010) Differential Expression of Maize Chitinases in the Presence or Absence of Trichoderma harzianum Strain T22 and Indications of a Novel Exo-Endo-Heterodimeric Chitinase Activity. BMC Plant Biology, 10, 136-146. http://dx.doi.org/10.1016/S0031-9422(02)00196-6

[16] Fujii, Y., Asahara, M., Ichinose, M. and Nakajima, H. (2002) Fungal Melanin Inhibitor and Related Compounds from Penicillium decumbens. Phytochemistry, 60, 703-708. http://dx.doi.org/10.1016/S0031-9422(02)00196-6

[17] Nicoletti, R. and Stefano, M.D. (2012) Penicillium restrictum as an Antagonist of Plant Pathogenic Fungi. Dynamic Biochemistry Process Biotechnology and Molecular Biology, 6, 61-69.

[18] Kim, J.C., Choi, G.J., Park, J.H., Kim, H.T. and Cho, K.Y. (2001) Activity against Plant Pathogenic Fungi of Phomalactone Isolated from Nigrospora sphaerica. Pest Management Science, 57, 554-559. http://dx.doi.org/10.1002/ps.318

[19] Tanaka, M., Fukushima, T., Tsujino, Y. and Fujimori, T. (1997) Nigrosporin A and B, New Phytotoxic and Antibacterial Metabolities Produced by a Fungus Nigrospora oryzae. Bioscience Biotechnology, and Biochemistry, 61, 18481852. http://dx.doi.org/10.1271/bbb.61.1848

[20] Whalley, A.J.S. and Edwards, R.L. (1995) Secondary Metabolites and Systematic Arrangement within the Xylariaceae. Canadian Journal of Botany, 73, 802-810. http://dx.doi.org/10.1139/b95-325 